Niina Hämäläinen : Yhteinen Perhe, Jaetut Tunteet. Lyyrisen kansanrunon tekstualisoinnin ja artikuloinnin tapoja Kalevalassa

[Une famille, des sentiments partagés. Modes de textualisation et d'articulation de la poésie populaire lyrique dans le Kalevala], Turku, Turun Yliopisto, 2012, 338 p.

\title{
Aleksi Moine
}

\section{(2) OpenEdition} Journals

Édition électronique

URL : https://journals.openedition.org/efo/5596

DOI : $10.4000 /$ efo. 5596

ISSN : 2275-1947

Éditeur

INALCO

Édition imprimée

ISBN : 978-2-343-08571-5

ISSN : 0071-2051

Référence électronique

Aleksi Moine, « Niina Hämäläinen : Yhteinen Perhe, Jaetut Tunteet. Lyyrisen kansanrunon tekstualisoinnin ja artikuloinnin tapoja Kalevalassa ", Études finno-ougriennes [En ligne], 47 | 2015, mis en ligne le 07 juillet 2016, consulté le 20 septembre 2021. URL : http://journals.openedition.org/efo/5596 ; DOI https://doi.org/10.4000/efo.5596

Ce document a été généré automatiquement le 20 septembre 2021.

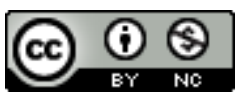

Études finno-ougriennes est mis à disposition selon les termes de la Licence Creative Commons Attribution - Pas d'Utilisation Commerciale 4.0 International. 


\section{Niina Hämäläinen : Yhteinen Perhe, Jaetut Tunteet. Lyyrisen kansanrunon tekstualisoinnin ja artikuloinnin tapoja Kalevalassa}

[Une famille, des sentiments partagés. Modes de textualisation et d'articulation de la poésie populaire lyrique dans le Kalevala], Turku, Turun Yliopisto, 2012, $338 \mathrm{p}$.

\section{Aleksi Moine}

\section{1 [Image non convertie]}

2 Cet ouvrage est le texte de la thèse de doctorat de Niina Hämäläinen, soutenue à l'université de Turku en 2012. Pouvant intéresser à la fois les folkloristes et les chercheurs en littérature, cette thèse brillante permet d'éclairer les rapports entre la poésie populaire finnoise et l'épopée du Kalevala, considérée comme œuvre littéraire.

3 Pour ce faire, l'auteure adopte en particulier la perspective de la poésie lyrique, qui a été quelque peu négligée par la recherche. L'ouvrage est structuré en quatre parties et neuf chapitres. Les deux premières parties constituent en quelque sorte une longue introduction qui permet de construire le cadre conceptuel qui servira de base théorique aux analyses de Hämäläinen. Dans la première, l'auteure pose ainsi les fondements théoriques de sa réflexion, tandis que la seconde constitue une cartographie du contexte, tant littéraire et culturel qu'historique et social, dans lequel le Kalevala a été créé. La troisième partie (chapitres 5 à 7) propose une analyse thématique de la poésie populaire lyrique et de sa textualisation dans l'épopée ; les deux derniers chapitres font office de conclusion et rassemblent clairement les réflexions développées dans l'ensemble de l'ouvrage.

4 Les deux concepts clés de l'analyse de Hämäläinen sont ceux de textualisation et d'articulation. Ces concepts sont très précisément définis et interrogés dans l'introduction, mais il peut être intéressant d'en dire quelques mots, sans bien sûr prétendre entrer dans les détails de l'analyse. La textualisation désigne les différents 
objectifs et procédés qui entrent en jeu dans le processus de mise à l'écrit de la poésie populaire lyrique, fondamentalement orale. Le concept d'articulation permet d'affiner la définition de la textualisation, en montrant comment l'activité d'écriture de Lönnrot s'insère dans un cadre historique et culturel, comme un pont qui permet de relier, d'articuler différentes classes de la société et différents types de phénomènes culturels. Hämäläinen part ainsi de l'idée de Honko que l'épopée de Lönnrot peut être analysée de la même manière qu'une épopée orale. C'est aussi pourquoi elle étudie les cinq versions différentes du texte qui ont été publiées par Lönnrot : elles constituent précisément le corpus dense, préconisé par la méthode d'analyse de Honko. Outre ces cinq textes écrits, l'analyse porte sur le corpus de poésie populaire recueilli, transcrit par Lönnrot ou par d'autres - mais dans ce cas, seulement les textes auxquels il a pu avoir accès. La thèse de Hämäläinen se révèle un dialogue particulièrement bien construit entre ces différents corpus et s'appuie également sur les textes plus théoriques de Lönnrot, notamment ses préfaces ou ses lettres: la comparaison précise des textes permet de mettre en lumière les intentions et les objectifs qu'avait Lönnrot lorsqu'il rédigeait son épopée. Cette comparaison représente, de la part de l'auteure, une tentative de faire entendre les différentes voix qui ont pu entrer en dialogue entre elles au XIX ${ }^{\mathrm{e}}$ siècle, des voix appartenant à différentes classes sociales et exprimant des opinions différentes sur les sentiments et sur la structure familiale. L'étude est centrée sur les deux personnages d'Aino et de Kullervo: ces deux épisodes sont en effet ceux qui contiennent le plus de vers lyriques et qui sont sans doute les plus représentatifs en ce qui concerne l'expression des sentiments. Le choix de traiter de la poésie lyrique, en particulier des huolirunot (les chants de peine), est lié au rôle particulier qu'elle joue dans la conception qu'a Lönnrot de la poésie populaire : la poésie lyrique occupe une place d'intermédiaire entre le peuple et la bourgeoisie cultivée qui constitue le lectorat de Lönnrot et ne connaît pas les formes traditionnelles de la poésie populaire. La poésie lyrique devient, dans l'épopée, comme un métadiscours, un discours direct, qui permet à Lönnrot de montrer du doigt à son public ce qu'est la poésie populaire.

En s'appuyant sur les réflexions sur la théorie de la réception et l'horizon d'attente des lecteurs, l'auteure renouvelle ainsi la perception du Kalevala, et en particulier de la façon d'envisager la poésie lyrique qui est insérée dans l'épopée. Elle montre que l'insertion de ces vers lyriques n'a pas seulement une importance dans la sphère littéraire, et dans la définition de la poésie populaire auprès du lectorat de Lönnrot, mais qu'elle se fait aussi le reflet de discussions contemporaines sur la famille, notamment sur le rôle de la mère - problématiques qui sont davantage celles des classes aisées de la société que du milieu rural où ont été recueillis les poèmes.

6 La précision de l'analyse textuelle de Hämäläinen rend bien compte de l'évolution de la pensée de Lönnrot, et de son rapport à la poésie populaire. La contextualisation historique permet de voir comment se mêlent les deux voix, archaïque et moderne, au sein de l'épopée de Lönnrot, qui devient de cette façon une épopée moderne, qu'il convient d'étudier au travers du concept d'articulation, comme un carrefour entre plusieurs dimensions, littéraires, mais aussi sociales. Par la clarté de son argumentation et par la profondeur et la précision de son analyse, la thèse de Niina Hämäläinen est un ouvrage particulièrement stimulant. Les questions qu'elle pose dépassent largement le cadre du Kalevala et peuvent sans nul doute ouvrir de nouvelles perspectives dans d'autres domaines que les études de folklore. Cet ouvrage pourra ainsi nourrir de façon 
fructueuse tous ceux qui s'intéressent à la poésie populaire, à la littérature, mais aussi à l'histoire sociale et à la construction des modèles familiaux.

\section{INDEX}

Index géographique : Finlande, Turku

Keywords : Folk Poetry, Lyrical Poetry, Textualisation, Reception

Mots-clés : poésie populaire, poésie lyrique, textualisation, réception

motscleset rahvaluule, lüüriline luule, tekstualiseerimine, retseptsioon.

motsclesru НАРОДНАЯ ПОЭЗИЯ, ЛИРИЧЕСКАЯ ПОЭЗИЯ, ТЕКСТУАЛИЗАЦИЯ, РЕЦЕПЦИЯ.

nomsmotscles Finnois

Index chronologique : XIXe siècle

Thèmes : études littéraires, études de folklore 